\title{
Pain response in a population-based study of radium-223 (Ra223) for metastatic castration- resistant prostate cancer
}

Sunil Parimi ${ }^{1 *}$; Suraya Bondy ${ }^{2 *}$; Erica Tsang ${ }^{1}$; Michael Ross McKenzie ${ }^{3}$; Francois Bachand ${ }^{3}$; Maria Aparicio'; ${ }^{2}$ Graeme Duncan ${ }^{3}$; Katherine Sunderland ${ }^{2}$; Robert Anton Olson ${ }^{3}$; Howard Huaihan $\mathrm{Pai}^{3}$; Abraham Skaria Alexander ${ }^{3}$; Vincent LaPointe ${ }^{3}$; Kim Nguyen Chi ${ }^{1}$; Scott Tyldesley $^{3}$

${ }^{1}$ Medical Oncology, British Columbia Cancer Agency, BC, Canada; ${ }^{2}$ Genitourinary Cancer Outcomes Unit, British Columbia Cancer Agency, BC, Canada; ${ }^{3}$ Radiation Oncology, British Columbia Cancer Agency, BC, Canada

${ }^{*}$ Equal contribution

Cite as: Can Urol Assoc J 2019 February 7; Epub ahead of print. http://dx.doi.org/10.5489/cuaj.5685

Published online February 7, 2019

$* * *$

\section{Abstract}

Introduction: Clinical trials have shown that radium-223 (Ra223) can prolong survival and improve quality of life in patients with metastatic castration-resistant prostate cancer (mCRPC). The objectives of this study were to evaluate pain responses with Ra223 at a population-based level and to determine if there is an association between pain response and alkaline phosphatase (ALP) response.

Methods: All patients from the Vancouver and Kelowna Cancer Centres (CC) in British Columbia who were treated with Ra223 between June 2015 and December 2016 were identified. Patients completed the Brief Pain Inventory (BPI) just prior to each Ra223 injection. Pain response was defined as a two or more point improvement in worst pain relative to baseline, without an increase in pain medication level. ALP was determined at each visit, with a response threshold defined as a $30 \%$ decrease from baseline, consistent with the definition of response used in the ALSYMPCA trial.

Results: A total of 65 patients in Vancouver and Kelowna CC received Ra223 during the study period and 56 patients had at least one BPI record, of which 44 (79\%) patients were assessable for change in worst pain. Of the assessable patients, 23 (52\%, 95\% confidence interval [CI] 3867) had a pain response, although the use of concurrent external beam radiotherapy was a confounder in four cases. Of the 44 patients assessable for change in worst pain, 59\% had ALP responses greater than $30 \%$. An ALP response was seen in $56 \%$ of pain-responders vs. $43 \%$ of 
non-pain-responders. There was no association between pain response and ALP response (Phi =0.05; p=0.77).

Conclusions: Ra223 administration was associated with a meaningful pain response rate in this cohort. There was no correlation between pain response and ALP response.

\section{Introduction}

Bone metastases from prostate cancer frequently cause bone pain. Ra223 is an alpha-emitting calcium mimetic that is incorporated into areas of active bone turnover and delivers alphaparticle radiation to the areas of active bone involvement in patients with prostate cancer. In the ALSYMPCA trial, Ra223 improved overall survival (OS), prolonged time to first symptomatic skeletal event (SSE) by 5.8 months (HR 0.66, 95\% CI 0.52-0.83), delayed time to opiate use and improved quality of life compared to placebo in patients with symptomatic bone metastases from castration resistant prostate cancer (CRPC). ${ }^{1}$ A phase II trial by Nilsson et al additionally demonstrated that $56 \%$ of patients with mCRPC demonstrated a pain response after a single injection of standard-dosage Ra223, where "pain response" was defined as a decrease on a pain

index comprised of a visual analog scale and analgesic usage. ${ }^{2}$ US Early Assess Program (EAP) analysis also reported a 59\% pain improvement of at least 2 points over baseline on BPI in eligible patients. ${ }^{3}$ Active bone turnover is associated with increased bone alkaline phosphatase (ALP) levels in the blood. OS benefit has been correlated with a decrease in ALP levels during Ra223 therapy; however, it is unclear whether a declining ALP level with Ra223 is also associated with improved pain.

The aim of this study was to evaluate baseline pain and pain response in prostate cancer patients treated with Ra223 in the general population of patients being treated for CRPC, and to ascertain whether pain response correlates with ALP response during Ra223 therapy.

\section{Methods}

BC Cancer is the single provider of Ra223 in British Columbia (BC), Canada. All treated cases are centrally registered. Each of the six regional cancer centres in BC has a defined populationbased catchment area. Data from all patients treated with Ra223 has been collected from the BC Cancer Provincial Radiation Therapy Program's electronic medical records. Starting in June 2015, patients treated with Ra223 who resided in the Kelowna and Vancouver CC catchment areas were asked to complete the Brief Pain Inventory (BPI) through an electronic patient reported outcomes (PRO) platform.

The PRO platform was used for data abstraction and charts were reviewed for data augmentation. All CRPC patients from the Vancouver and Kelowna Centre catchments who were prescribed 6 cycles of Ra223 therapy between June 2015 (when the PRO platform was activated for Ra223 patients) and December 2016 (to allow for minimum of 6 months follow-up) were included in the study. At each visit, patients completed PRO questionnaires which included 
the BPI. Patients were asked about pain medication use at each visit, which was classified as 1) no opioids, 2) weak opioids, or 3) strong opioids. A change between pain medication groups was categorized as increased, decreased or stable accordingly. Neither actual doses of opioids and morphine equivalent units nor daily opioid diaries were captured. Patients were also asked to rate their pain at its worst on a 10-point scale at each visit. Pain response was determined by calculating the degree of change in worst pain relative to baseline. Eight (20\%) patients did not have a baseline pain score prior to first dose of Ra223, and these cases were assessed for response using their pain score prior to their second dose of Ra223. In an effort to assess the impact of these patients on the response rate, we performed maximizing and minimizing sensitivity analyses. Patients who demonstrated a decrease in pain of at least 2 units from baseline and whose pain medication category decreased or remained stable were considered pain responders, using BPI pain response criteria described by Chow et al. ${ }^{4}$ An ALP level was also taken at each visit and analyzed for response. An ALP response was defined as a decrease of $30 \%$ or more from baseline values consistent with definition used in ALSYMPCA trial. Correlation between pain response and ALP responses was assessed using a Phi statistic. To further characterize the participants involved, a number of additional baseline factors were recorded, including age, Eastern Cooperative Oncology Group (ECOG) performance status, number of bone metastases, analgesic use, palliative radiotherapy to bone during the course of Ra223 and lines of therapy (including palliative radiotherapy to bone) prior to Ra223. Laboratory values such as levels of hemoglobin, albumin, lactate dehydrogenase (LDH), prostate specific antigen (PSA) and ALP before Ra223 injections were also assessed.

\section{Results}

In total, 65 patients from the Vancouver and Kelowna catchment areas were treated with Ra223 and 56 (86\%) of the patients from these centres had at least one BPI record. We were able to assess change in worst pain in 44 (79\%) of the patients (See Figure 1). Baseline factors for all 65 patients that received Ra223 (Ra223 group), the sub-group of 56 patients who had a PRO record (PRO group), and the $44 \mathrm{PRO}$ patients that were evaluable for pain response (Pain group) are presented in Table 1. The summary of Time 1 pain scores for the Pain group displayed in Table 1 includes cases where the pain score prior to the second dose of Ra223 was used in the absence of a true baseline value.

There were 39 of the 56 PRO-group patients who had a true baseline pain measure and $92 \%$ of these had at least some pain, $70 \%$ had a pain score of at least 3, and 31\% scored 7 or above (See Figure 2). Of the 44 patients that were assessable for change in worst pain, 36 had a true baseline pain measure. For the 8 patients that did not have a pain score prior to first dose, the baseline was the pain score prior to second dose. A sensitivity analysis was performed to show that the impact of having 8 patients without a documented pain score prior to first dose was small. Of those 8 patients, 2 exhibited a pain response and 6 did not. The reported percentage of responders was 52\% (95\%CI 38\%-67\%). Had all 6 cases been scored as pain responders, this 
would have changed the percentage of responders to 66\% (95\%CI 52\%-80\%). Had all 6 cases been scored as non-responders, this would have changed the percentage to 48\% (95\%CI 33\%$62 \%)$. Of note, all of the three confidence intervals overlapped in this analysis.

In total, 24 Pain group patients (55\%) showed an improvement of at least 2 units on worst pain score, and 23 (52\% of all Pain group patients, 95\%CI 38\%-67\%) had a pain response. Four (17\%) of the 23 pain responders also received palliative external beam radiation therapy (EBRT) during the course of Ra223 that could have impacted their pain response. Of those with pain score of 3 or higher at baseline, 65\% showed pain improvement of at least 2 units during the course of Ra223 treatment. Figure 3 shows the maximum decrease in worst pain score for the Pain group. Figure 4 plots the sequence of pain response/non-response from baseline for each patient, with an indicator for the timing of EBRT. Pain responses were often short-lived, and did not have a clear time pattern, although for 13 of 21 cases (62\%) with complete time records, the pain response was seen after the first dose.

Figure 5 plots the maximum decrease in ALP for each patient in the Pain group. ALP response measured in the 44 Pain group patients indicates that $59 \%$ of patients had an ALP response greater than $30 \%$. Comparison of pain response to ALP response showed that $56 \%$ of pain-responders had an ALP response and 43\% of non-pain-responders had an ALP response. ALP and pain responses were assessed using a Phi statistic, and ALP response was not significantly correlated to pain response ( $\mathrm{Phi}=-0.05, \mathrm{p}=0.77$ ).

As a secondary analysis, pain response was evaluated at any time during Ra223 therapy. In this case, pain response was determined by calculating the degree of change in worst pain relative to any worst pain score that occurred prior. In this secondary analysis, patients who demonstrated a decrease in pain of at least 2 units from any prior score and whose pain medication category decreased or remained stable for the relevant period were considered pain responders.

Results using this approach were similar to those comparing pain to baseline; 27 of the Pain group patients (61\%) showed an improvement of at least 2 units over the worst pain score at some point during the course of Ra223 therapy with stable or improved pain medication category. Four (17\%) of the 27 pain responders also received palliative external beam radiation therapy (EBRT) during the course of Ra223 that could have impacted their pain response. Comparing pain response at any time to ALP response indicates that $59 \%$ of pain-responders had an ALP response and $41 \%$ of non-pain-responders had an ALP response. ALP response at any time was not significantly correlated to pain response $(\mathrm{Phi}=0.004, \mathrm{p}=1.00)$.

\section{Discussion}

A baseline pain level of 3 or higher was present in $70 \%$ of patients, and $52 \%$ of evaluable patients had a pain response. This finding is consistent with the findings in other studies of a longer median time to initial opioid use for Ra223 compared to placebo (HR 0.621, 95\%CI 0.460.85), as well as a longer time to external beam radiation therapy (EBRT) for bone pain (HR 
0.67, 95\%CI 0.53-0.85), and prolonged time to first symptomatic skeletal event (HR 0.66, 95\%CI 0.52-0.83) (defined as first use of EBRT for bone pain, new symptomatic pathologic fracture, spinal cord compression, or cancer-related orthopedic surgery intervention). ${ }^{1,5}$ Clinical trials patients tend to have more favorable outcomes than those treated in non-trial settings, often due to the rigorous eligibility criteria that have to be met. Population-based data increases the generalizability of results by reflecting a broader range of patient experiences, institutional practices, and physician expertise. ${ }^{6,7}$ Our analysis provides such population-based data and is consistent with the pain improvement rate seen with Ra223 in the US EAP program data. ${ }^{3}$ As ALP levels can reflect bone activity, we postulated that the pain response during Ra223 treatment may correlate with ALP response. Our study failed to show such a relationship, which is the second major finding of this paper. This suggests that ALP levels alone do not reflect the pain experienced by patients with CRPC experience during Ra223. It is also possible that our study was too small to see a correlation, and further work in this area should continue. Ra223 has an effect on tumor cells within the bone, surrounding osteoblasts, ${ }^{8}$ and the cancer microenvironment. ${ }^{9-11}$ Any or all of these mechanisms could contribute to pain relief yet not all would lead to a decline in ALP, which is primarily a marker of osteoblastic activity in this context. ${ }^{12}$ Our data suggest that neither the ALP response, nor the absence of response, gives the treating physician an indication of a lack of eventual pain response in an individual patient. This study is limited by small patient numbers, its retrospective nature, and that not all patients are willing and able to complete a BPI form at every visit in a population-based setting. The characterization of pain responses, while informative, must also be scrutinized. An analgesic response may have been augmented due to the use of EBRT during the trial period, although only 4 of the 23 patients characterized as eligible responders in our analysis had EBRT during the course of Ra223 that could have affected the pain response. It is also possible that pain responses could have been over- or under-estimated, as under-reporting of pain by cancer patients is a common issue. ${ }^{13}$ It must also be acknowledged that increases or decreases in opiate medications could have taken place once these agents were started, which was not fully assessed and could confound the true nature of changes in pain, as the BPI tool used at monthly intervals do not capture pain medication use in a way that allows for conversion to morphine equivalent units. The role of PRO measures in our institutions is evolving and will be increasingly used to identify and address symptom burden in the future. We did not collect the use of bone-protecting agents (such as bisphosphonates or RANK-L inhibitors), which could have meaningful impacts on the bone-pain. ${ }^{14-16}$ However, such agents are rarely used in BC for patients with prostate cancer.

Future studies would be helpful to explore baseline clinical factors that predict analgesic benefit from Ra223. Studies are now also exploring Ra223 administered with more contemporary mCRPC agents. ${ }^{17-18}$ Future studies of the relationship between ALP response and 
pain response during Ra223 should include more patients and ideally daily pain medication logs to allow for more accurate pain assessment.

\section{Conclusion}

This population-based analysis suggests that meaningful pain responses are observed during a course of Ra223 in a population-based setting, although the changes are difficult to attribute entirely to the use of Ra223. While ALP levels declined in many cases during a course of Ra223, as expected, there was no observed correlation identified between pain response and ALP response. 


\section{References}

1. Parker C, Nilsson S, Heinrich D, et al. Alpha emitter radium-223 and survival in metastatic prostate cancer. N Engl J Med 2013;369:213-23.

2. Nilsson S, Strang P, Aksnes AK, et al. A randomized, dose-response, multicenter phase II study of radium-223 chloride for the palliation of painful bone metastases in patients with castration-resistant prostate cancer. Eur J Cancer 2012;48(5):678-86.

3. Morris MJ, Sartor AO, Vogelzang NJ, et al. Effect of radium-223 dichloride (Ra-223) on pain from US EAP. J Clin Oncol. 33, 2015 (suppl 7; abstr 160).

4. Chow E, James J, Barsevick A et al. Confirmatory factor analysis of brief pain inventory (BPI) functional interference clusters in patients with bone metastases. J Pain Manag. 2010; 3(3): 247-53.

5. Sartor O, Coleman R, Nilsson S, et al. Effect of radium-223 dichloride on symptomatic skeletal events in patients with castration-resistant prostate cancer and bone metastases: results from a phase 3, double-blind, randomised trial. Lancet Oncol 2014;15:738-46.

6. Layde PM, Broste SK, Desbiens N, et al. Generalizability of clinical studies conducted at tertiary care medical centers: a population-based analysis. J Clin Epidemiol 1996;49(8):835-41.

7. Yusuf S, Collins R, and Peto R. Why do we need some large, simple randomized trials? Stat Med 1984;3:409-20.

8. Heinrich D, Bektic J, Bergman A, et al. The contemporary use of radium-223 in metastatic castration-resistant prostate cancer. Clin Genitourin Cancer 2017; [epub ahead of print].

9. Henriksen G, Breistol K, Bruland OS, et al. Significant antitumor effect from boneseeking, alpha-particle-emitting (223)Ra demonstrated in an experimental skeletal metastases model. Cancer Res 2002;62:3120-5.

10. Bruland OS, Nilsson S, Fisher DR, et al. High-linear energy transfer irradiation targeted to skeletal metastases by the alpha-emitter 223Ra: adjuvant or alternative to conventional modalities? Clin Cancer Res 2006;12:6250s-7s.

11. Suominen MI, Fagerlund KM, Rissanen JP, et al. Radium-223 inhibits osseous prostate cancer growth by dual targeting of cancer cells and bone microenvironment in mouse models. Clin Cancer Res 2017;23:4335-46.

12. Nome R, Hernes E, Bogsrud TV, et al. Changes in prostate-specific antigen, markers of bone metabolism and bone scans after treatment with radium-223. Scand J Urol 2015;49(3):211-7.

13. Sprangers MA and Aaronson NK. The role of health care providers and significant others in evaluating the quality of life of patients with chronic disease: a review. J Clin Epidemiol 1992;45:743-60. 
14. Saad F, Gleason DM, Murray R, et al. A randomized, placebo-controlled trial of zoledronic acid in patients with hormone-refractory metastatic prostate carcinoma. $\mathrm{J} \mathrm{Natl}$ Cancer Inst 2002;94(19):1458-68.

15. Rodrigues P, Hering F, and Campagnari JC. Use of bisphosphonates can dramatically improve pain in advanced hormone-refractory prostate cancer patients. Prostate Cancer Prostatic Dis 2004;7:350-4.

16. Fizazi K, Carducci M, Smith M, et al. Denosumab versus zoledronic acid for treatment of bone metastases in men with castration-resistant prostate cancer: a randomised, doubleblind study. Lancet 2011;377(9768):813-22.

17. Saad F, Carles J, Gillessen S, et al. Radium-223 and concomitant therapies in patients with metastatic castration-resistant prostate cancer: an international, early access, openlabel, single-arm phase 3b trial. Lancet Oncol 2016;17(9):1306-16.

18. Sartor O, Vogelzang NJ, Sweeney C, et al. Radium-223 safety, efficacy, and concurrent use with abiraterone or enzalutamide: first U.S. experience from an Expanded Access Program. Oncologist 2017; [epub ahead of print]. 


\section{Figures and Tables}

Fig. 1. Consort diagram.

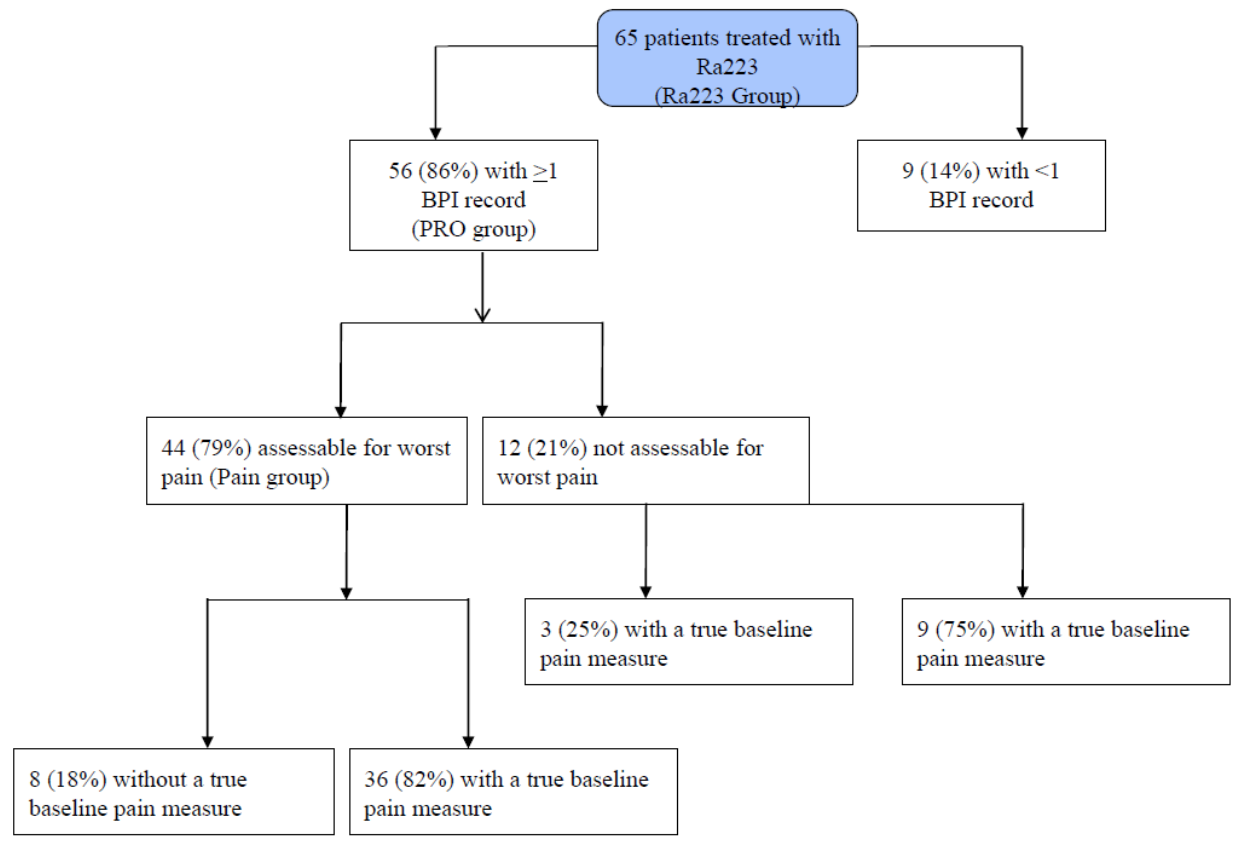

Fig. 2. Worst pain score for patients with a true baseline (on or up to 35 days before the final injection) in the PRO group $(n=39)$.

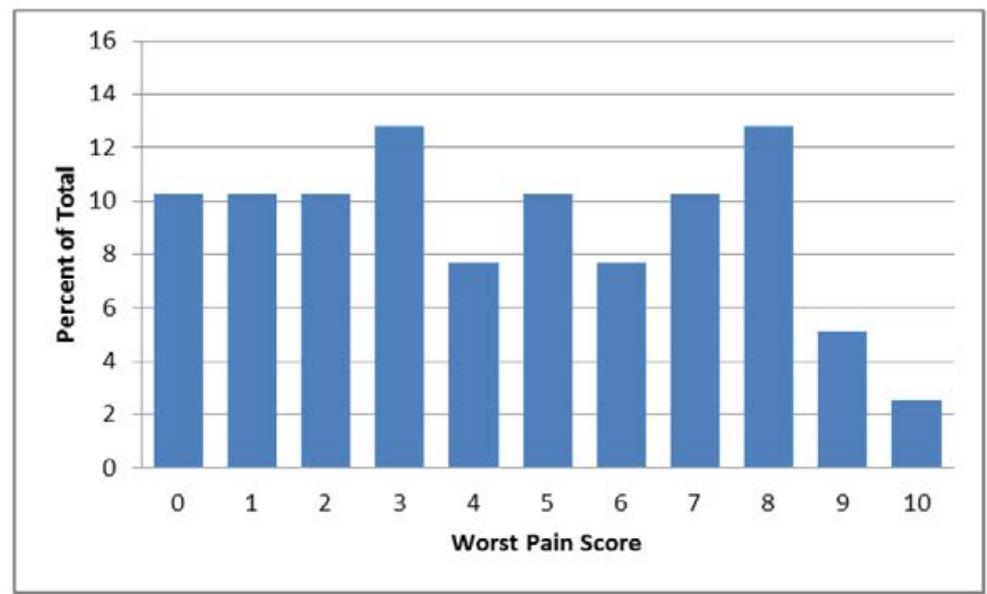


Fig. 3. Waterfall plot of maximum decrease in worst pain for the Pain group ( $n=44)$.

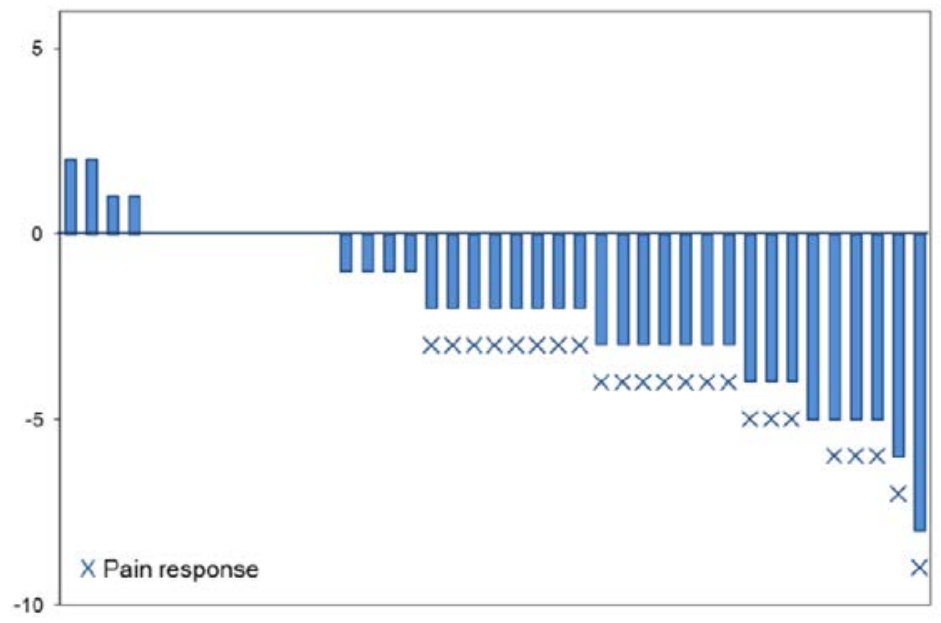

Fig. 4. Sequence of pain response/non-response from baseline by patient in the Pain group $(n=44)$.

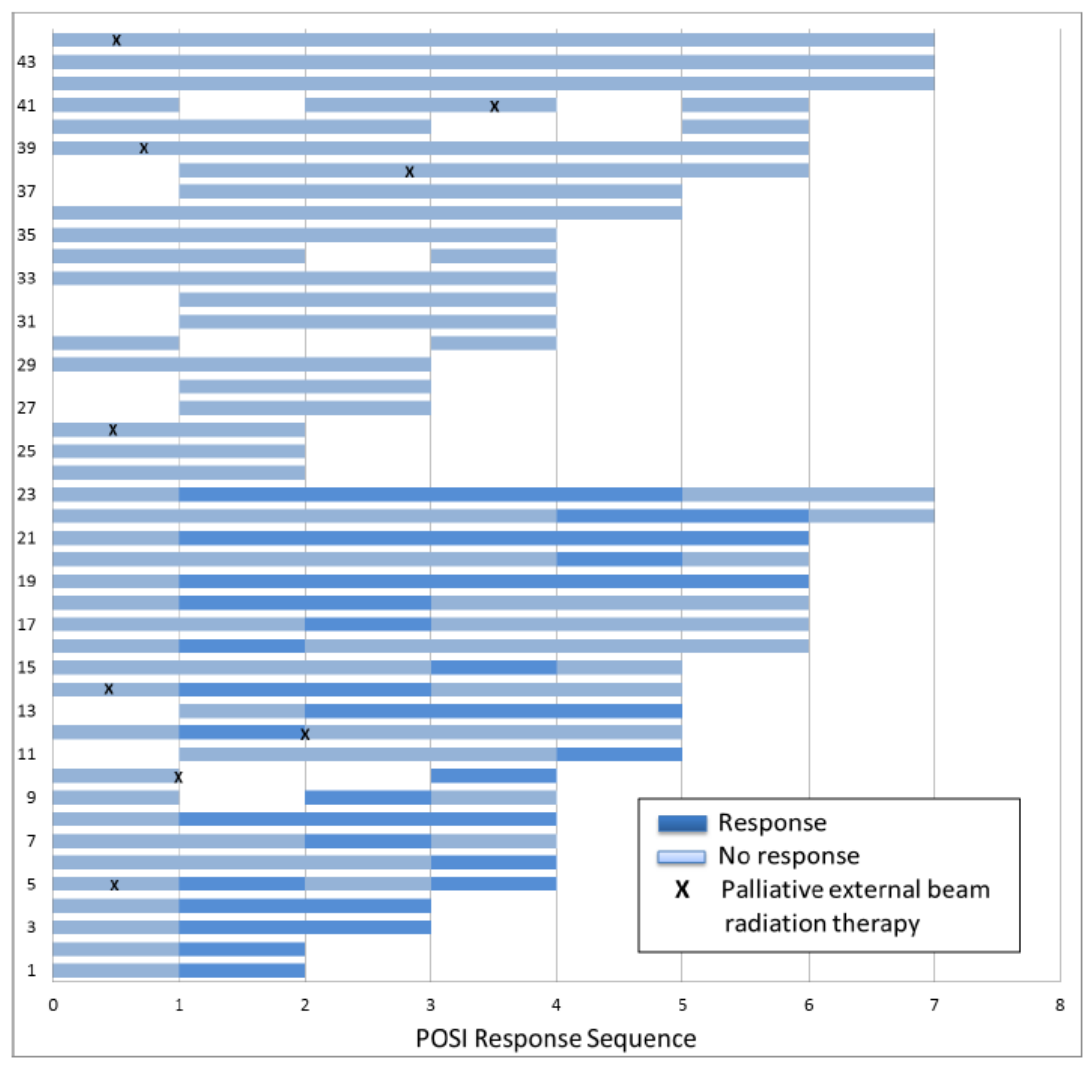


Fig. 5. Waterfall plot of the maximum alkaline phosphatase decline for each patient in the Pain group $(\mathrm{n}=44)$.

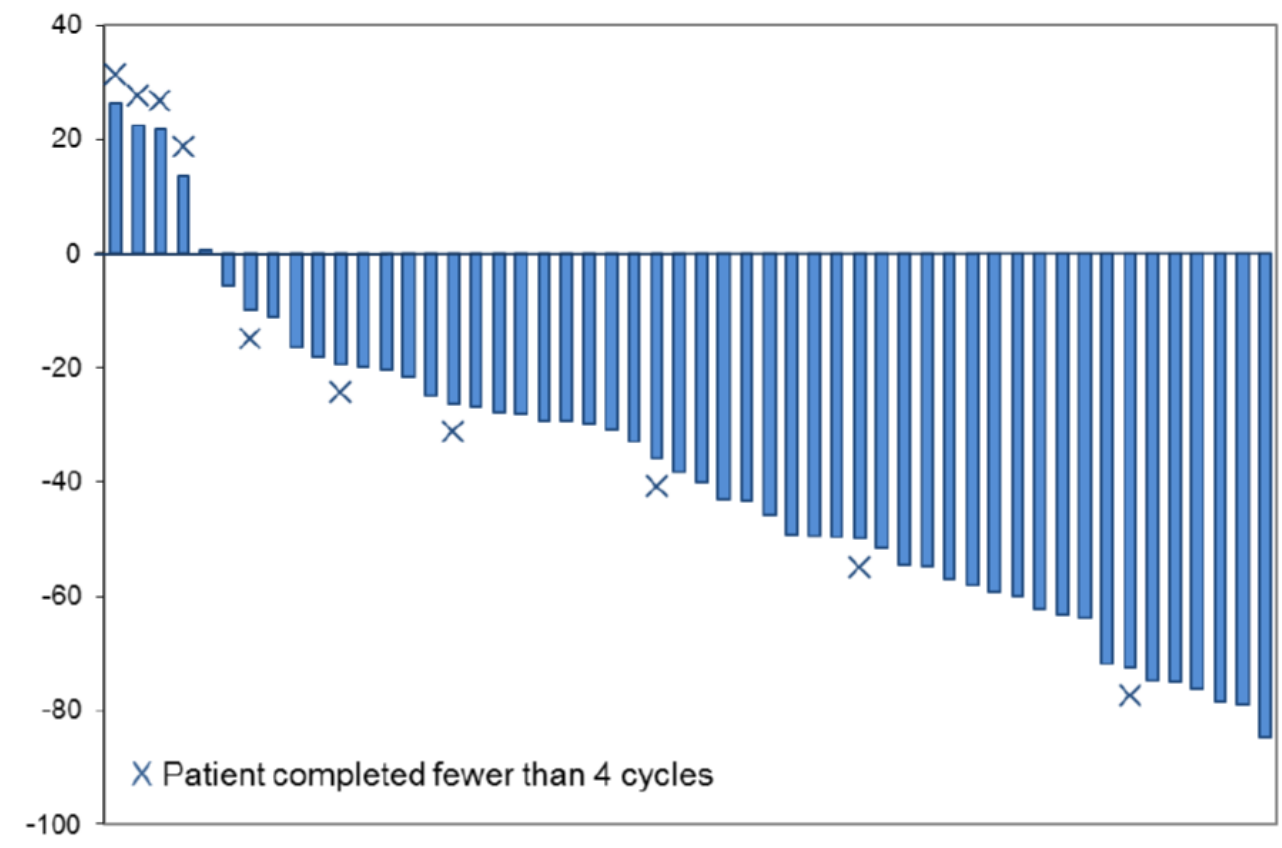


Table 1. Baseline characteristics

\begin{tabular}{|c|c|c|c|}
\hline & Ra223 group & PRO group & Pain group \\
\hline & $(n=65)$ & $(n=56)$ & $(n=44)$ \\
\hline Age, median (range) & 76 (57-94) & $76(57-94)$ & 74 (59-94) \\
\hline \multicolumn{4}{|l|}{ ECOG } \\
\hline$<2, \mathrm{n}(\%)$ & $48(74)$ & $45(80)$ & $35(80)$ \\
\hline$\geq 2, \mathrm{n}(\%)$ & $17(26)$ & $11(20)$ & $9(20)$ \\
\hline \multicolumn{4}{|l|}{ Bone mets } \\
\hline$<6, \mathrm{n}(\%)$ & $13(20)$ & $8(15)$ & $8(18)$ \\
\hline$\geq 6, \mathrm{n}(\%)$ & $52(80)$ & $45(85)$ & $36(82)$ \\
\hline HB, median (range) & $125(94-146)$ & $123(94-146)$ & $124(98-146)$ \\
\hline ALP, median (range) & $148(27-1294)$ & $148(27-1294)$ & $144(27-1294)$ \\
\hline Albumin, median (range) & $40(20.6-99)$ & 40 (20.6-99) & 40 (27-99) \\
\hline LDH, median (range) & $295(130-2115)$ & $345(130-2115)$ & 337.5 (130-2115) \\
\hline PSA, median (range) & $84.88(0.24-2800)$ & $98.7(0.24-2800)$ & $99.215(0.24-2800)$ \\
\hline $\begin{array}{l}\text { Previous systemic } \\
\text { treatments, median } \\
\text { (range) }\end{array}$ & $2(0-5)$ & $2(0-5)$ & $2(0-5)$ \\
\hline Docetaxel, n (\%) & $25(38)$ & $19(36)$ & $15(34)$ \\
\hline Abiraterone, n (\%) & $43(66)$ & $37(70)$ & $32(73)$ \\
\hline Enzalutamide, n (\%) & $47(72)$ & $36(68)$ & $29(66)$ \\
\hline Cabazitaxel, n (\%) & $4(6)$ & $2(4)$ & $2(5)$ \\
\hline $\begin{array}{l}\text { Previous clinical trial, } n \\
(\%)\end{array}$ & 25 (39) & $19(37)$ & $16(36)$ \\
\hline $\begin{array}{l}\text { Baseline pain "' median } \\
\text { (range) }\end{array}$ & & & $4(0-10)$ \\
\hline \multicolumn{4}{|l|}{ Baseline pain medication $^{*}$} \\
\hline None, n (\%) & & & $13(29.5)$ \\
\hline Non-opioids, n (\%) & 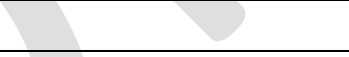 & & $10(23)$ \\
\hline Weak opioids, n (\%) & & & $8(18)$ \\
\hline Strong opioids, n (\%) & & & $13(29.5)$ \\
\hline
\end{tabular}

*Includes cases where the pain score prior to the second dose of Ra223 was used in the absence of a true baseline value. ALP: alkaline phosphatase; ECOG: Eastern Cooperative Oncology Group; HB: hemoglobin; LDH: lactate dehydrogenase; PSA: prostate-specific antigen. 